\title{
Group Rings, $G$-Codes and Constructions of Self-Dual and Formally Self-Dual Codes
}

\author{
Steven T. Dougherty \\ Department of Mathematics \\ University of Scranton \\ Scranton, PA 18510 \\ USA \\ Joseph Gildea \\ Rhian Taylor \\ University of Chester \\ Chester, UK \\ Alexander Tylyshchak \\ Department of Algebra \\ Uzhgorod State University \\ Ukraine
}

September 10, 2017

\begin{abstract}
We describe $G$-codes, which are codes that are ideals in a group ring, where the ring is a finite commutative Frobenius ring and $G$ is an arbitrary finite group. We prove that the dual of a $G$-code is also a $G$-code. We give constructions of self-dual and formally self-dual codes in this setting and we improve the existing construction given in [13] by showing that one of the conditions given in the theorem is unnecessary and, moreover, it restricts the number of self-dual codes obtained by the construction. We show that several of the standard constructions of self-dual codes are found within our general framework. We prove that our constructed codes must have an automorphism group that contains $G$ as a subgroup. We also prove that a common construction technique for producing self-dual codes cannot produce the putative $[72,36,16]$ Type II code. Additionally, we show precisely which groups can be used to construct the extremal
\end{abstract}


Type II codes over length 24 and 48. We define quasi- $G$ codes and give a construction of these codes.

Key Words: Group rings; self-dual codes; codes over rings.

\section{Introduction}

Cyclic codes are characterized by the fact that the cyclic shift of any element in the code is an element in the code. These codes are one of the most widely studied families of codes. This is due, for the most part, to the fact that cyclic codes have an algebraic description as ideals in the polynomial ring $R[x] /\left\langle x^{n}-1\right\rangle$ where $R$ is a Frobenius ring and $n$ is the length of the code. To classify cyclic codes, it is simply a matter of finding ideals in this ring via a factorization of $x^{n}-1$ over $R$.

One of the key results about cyclic codes is that the dual code of a cyclic code is a cyclic code. This result allows for the complete study of cyclic codes to be done in the canonical algebraic setting. Cyclic codes have also been generalized in numerous ways, specifically to constacylic and negacyclic codes where $x^{n}-1$ is replaced with $x^{n}-\lambda$ for constacylic codes and $x^{n}+1$ for negacylic codes (that is $\lambda=-1$ ).

An alternate view of cyclic codes is to see them as ideals in the group ring $R C_{n}$ where $C_{n}$ is the cyclic group of order $n$. In this paper, we will study codes as ideals in an arbitrary group ring $R G$. This allows for an algebraic description of these codes as well as ensuring that the codes have a given group in their automorphism group. For very early work in this direction, see two papers by F.J. MacWilliams, [15] and [16]. We shall refer to codes that are ideals in the group ring $R G$ as $G$-codes. We shall prove here that, like cyclic codes, the dual of a $G$-code is again a $G$-code.

Quasi-cyclic codes are another generalization of cyclic codes. The codes have received less attention largely because they do not have a canonical representation in an algebraic setting. We make a generalization of this concept to quasi- $G$ cyclic codes. We give a construction, like that in $[6]$ for cyclic codes, for quasi- $G$ cyclic codes.

Self-dual codes over fields and rings are one of the most important and widely studied families of codes. They have interesting connections to groups, designs, lattices and other objects as well. As such, constructions of interesting self-dual codes are an important area of study in coding theory. In [13], Hurley gave a construction of self-dual codes from elements in a group algebra. The constructions were done generally in the group algebra $\mathbb{F}_{2} D_{2 k}$, where $D_{2 k}$ is the dihedral group of order $2 k$. In [18], McLoughlin gave a construction of the extremal $[48,24,12]$ using this construction technique. Additionally, numerous techniques have been described using commutative Frobenius rings to construct binary self-dual and formally self-dual codes by Yildiz, Karadeniz and others (see [9], [10], [11] for example). 
In this paper, we expand this construction to codes over finite commutative Frobenius rings and show how to construct isodual and formally self-dual codes as well. Additionally, we construct self-dual and formally self-dual codes over various families of rings, which, in turn, give formally self-dual and self-dual binary codes via a Gray map. We consider additional groups as well and expand the constructions using these groups.

\subsection{Codes}

The alphabet that we shall use for our codes is a finite commutative ring. It is also possible to study codes over non-commutative rings as well but we shall restrict ourselves to commutative rings in this paper. As such, from this point on we assume that all rings are commutative. Since the MacWilliams relations, which are fundamental in coding theory, only hold over Frobenius rings, we restrict ourselves to finite commutative Frobenius rings. For a description of coding theory in this setting see [5].

We begin by giving a characterization of Frobenius rings. Let $R$ be a finite ring. We assume that all rings contain a multiplicative identity. Let $\widehat{R}$ be the character module of the ring $R$. Then for a finite ring $R$ the following are equivalent.

- $R$ is a Frobenius ring.

- As a left module, $\widehat{R} \cong{ }_{R} R$.

- As a right module, $\widehat{R} \cong R_{R}$.

For commutative rings we can say that the $R$-module $R$ is injective and that if $R$ is a finite local ring with maximal ideal $\mathfrak{m}$ and residue field $\mathbf{k}$, then a Frobenius ring has $\operatorname{dim}_{\mathbf{k}} \operatorname{Ann}(\mathfrak{m})=$ 1. All of the rings used as alphabets in this paper will be assumed to be finite, commutative and Frobenius.

A code over $R$ of length $n$ is a subset of $R^{n}$. If the code is a submodule of $R^{n}$, then we say that the code is a linear code. We attach to the ambient space the usual inner-product, namely $[\mathbf{v}, \mathbf{w}]=\sum v_{i} w_{i}$ and define the orthogonal with respect to this inner-product as

$$
C^{\perp}=\left\{\mathbf{v} \in R^{n} \mid[\mathbf{v}, \mathbf{w}]=0, \forall \mathbf{w} \in C\right\}
$$

There is a unique orthogonal code because the ring is commutative. In the non-commutative case, there is both a left and right orthogonal. A code is said to be self-orthogonal if $C \subseteq C^{\perp}$ and self-dual if $C=C^{\perp}$. We say that two codes $C$ and $C^{\prime}$ are equivalent if $C^{\prime}$ can be formed from $C$ by permuting the coordinates of $C$. In some works about codes over rings, multiplication of a coordinate by a unit is allowed when defining equivalence, but note that we only allow permutation of coordinates in our definition of equivalence. A code $C$ is said to be isodual if $C$ and $C^{\perp}$ are equivalent codes. The automorphism group of a code $C$, 
denoted $\operatorname{Aut}(G)$, consists of all permutations of the coordinates of the code that fix the code.

Let $C$ be a code over a ring $R=\left\{a_{0}, a_{1}, \ldots, a_{r-1}\right\}$. The complete weight enumerator for the code $C$ is defined as:

$$
c w e_{C}\left(x_{a_{0}}, x_{a_{1}}, \ldots, x_{a_{r-1}}\right)=\sum_{\mathbf{c} \in C} \prod_{i=0}^{r-1} x_{a_{i}}^{n_{i}(\mathbf{c})}
$$

where there are $n_{i}(\mathbf{c})$ occurrences of $a_{i}$ in the vector $\mathbf{c}$.

The Hamming weight of a vector $\mathbf{v} \in R^{n}$ is $w t_{H}(\mathbf{v})=\left|\left\{i \mid v_{i} \neq 0\right\}\right|$. The Hamming weight enumerator is given by

$$
W_{C}(x, y)=\sum_{\mathbf{c} \in C} x^{n-w t_{H}(\mathbf{c})} y^{w t_{H}(\mathbf{c})}=c w e_{C}(x, y, y, \ldots, y)
$$

We say that a code is formally self-dual with respect to some weight enumerator if the code and its orthogonal have the same weight enumerator. It is possible for a code to be formally self-dual with respect to one weight enumerator and not another. For example, many codes are formally self-dual with respect to the Hamming weight enumerator without being formally self-dual with respect to the complete weight enumerator. Note that a selfdual code is also necessarily formally self-dual with respect to all weight enumerators.

Other weight enumerators are also possible, such as the symmetric weight enumerator or the Lee weight enumerator (which will be defined later) for specific rings. Since we only allow permutation of coordinates in our definition of equivalence, we have that if $C$ is isodual, then any weight enumerator for the code $C$ (complete, Hamming, symmetric, etc.) is identical to the weight enumerator of its orthogonal. This implies the following lemma which we will use in our constructions.

Lemma 1.1. If $C$ is an isodual code then it is formally self-dual with respect to any weight enumerator.

As mentioned before, we restrict ourselves to Frobenius rings since this is the class of rings for which MacWilliams relations exist. That is, the weight enumerator of a code over a Frobenius ring uniquely determines the weight enumerator of its orthogonal. See [5] for a complete description of these results. The MacWilliams relations imply that for a code $C$ over a Frobenius ring $R$ we have $|C|\left|C^{\perp}\right|=|R|^{n}$. This often fails for codes over nonFrobenius rings. In that sense, it is very difficult to discuss self-dual and formally self-dual codes over non-Frobenius rings. Moreover, this provides an easy way to prove that a ring is not Frobenius, that is, simply find an ideal $I$ whose annihilator does not have cardinality $|R| /|I|$.

A Gray map is a distance preserving map $\phi$ from $R$ to $\mathbb{F}_{2}^{t}$ for some $t$. We define the Lee weight, $w t_{L}(a)$ of an element $a \in R$ as the Hamming weight of $\phi(a)$. We then extend this to 
$R^{n}$ by saying that the Lee weight of a vector is the sum of the Lee weights of the coordinates of the vector. Then the Lee weight enumerator of a code $C$ over $R$ with an associated Gray map is defined as:

$$
L_{C}(x, y)=\sum_{\mathbf{c} \in C} x^{N-w t_{L}(\mathbf{c})} y^{w t_{L}(\mathbf{c})},
$$

where $N$ is the length of the binary image of the code $C$ under the Gray map. Note that the Lee weight enumerator of a code $C$ is the Hamming weight enumerator of the code $\phi(C)$.

\subsection{Group Rings}

We shall consider codes that are ideals inside of a group ring, where the ring is the alphabet of the code. We continue by giving the necessary definitions for group rings. Let $G$ be a finite group or order $n$, then the group $\operatorname{ring} R G$ consists of $\sum_{i=1}^{n} \alpha_{i} g_{i}, \alpha_{i} \in R, g_{i} \in G$.

Addition in the group ring is done by coordinate addition, namely

$$
\sum_{i=1}^{n} \alpha_{i} g_{i}+\sum_{i=1}^{n} \beta_{i} g_{i}=\sum_{i=1}^{n}\left(\alpha_{i}+\beta_{i}\right) g_{i}
$$

The product of two elements in a group ring is given by

$$
\left(\sum_{i=1}^{n} \alpha_{i} g_{i}\right)\left(\sum_{j=1}^{n} \beta_{j} g_{j}\right)=\sum_{i, j} \alpha_{i} \beta_{j} g_{i} g_{j}
$$

This gives that the coefficient of $g_{i}$ in the product is $\sum_{g_{i} g_{j}=g_{k}} \alpha_{i} \beta_{j}$.

Group rings are defined for groups and rings of arbitrary cardinality but, in this paper, we shall only be concerned with finite rings and finite groups since our alphabet for codes is a finite ring and codes are defined for finite length which corresponds to the size of the group. If $R$ is a field then the term group algebra is usually used in this case since the structure is an algebra as well. Throughout this paper we use $e_{G}$ to refer to the identity element of any group $G$.

We denote the space of $n$ by $n$ matrices with coefficients in $R$ by $M_{n}(R)$. Note that $M_{n}(R)$ is, in general, a non-commutative ring since multiplication of matrices is not commutative.

A matrix $M$, where the indices are given by the elements in $\mathbb{Z}_{n}$, is said to be circulant if $M_{i, j}=M_{1, j-i(\bmod n)}$, that is the matrix is formed by cycling the first row to the right. A matrix $M$, where the indices are given by the elements in $\mathbb{Z}_{n}$, is said to be reverse circulant if $M_{i, j}=M_{1, j+i(\bmod n)}$, that is the matrix is formed by cycling the first row to the left. It is immediately clear from the definition that a reverse circulant matrix is symmetric, that is, $M=M^{T}$. 


\section{Matrix Construction}

In this section, we shall give a construction of codes in $R^{n}$ from the group ring $R G$. This construction was first given for codes over fields by Hurley in [13]. Let $R$ be a finite commutative Frobenius ring and let $G=\left\{g_{1}, g_{2}, \ldots, g_{n}\right\}$ be a group of order $n$. Let $v=\alpha_{g_{1}} g_{1}+\alpha_{g_{2}} g_{2}+\cdots+\alpha_{g_{n}} g_{n} \in R G$. Define the matrix $\sigma(v) \in M_{n}(R)$ to be

$$
\sigma(v)=\left(\begin{array}{ccccc}
\alpha_{g_{1}^{-1} g_{1}} & \alpha_{g_{1}^{-1} g_{2}} & \alpha_{g_{1}^{-1} g_{3}} & \ldots & \alpha_{g_{1}^{-1} g_{n}} \\
\alpha_{g_{2}^{-1} g_{1}} & \alpha_{g_{2}^{-1} g_{2}} & \alpha_{g_{2}^{-1} g_{3}} & \ldots & \alpha_{g_{2}^{-1} g_{n}} \\
\vdots & \vdots & \vdots & \vdots & \vdots \\
\alpha_{g_{n}^{-1} g_{1}} & \alpha_{g_{n}^{-1} g_{2}} & \alpha_{g_{n}^{-1} g_{3}} & \ldots & \alpha_{g_{n}^{-1} g_{n}}
\end{array}\right) .
$$

The elements $g_{1}^{-1}, g_{2}^{-1}, \ldots, g_{n}^{-1}$ are simply the elements of the group $G$ in some order. We take this as the ordering of the elements since it makes the constructions more natural.

For a given element $v \in R G$, we define the following code over the ring $R$ :

$$
C(v)=\langle\sigma(v)\rangle .
$$

Namely, the code is formed by taking the row space of $\sigma(v)$ over the ring $R$. The code $C(v)$ is a linear code since it is the row space of a generator matrix, but it is not possible to determine the size of the code (or the dimension if $R$ is a field) immediately from the matrix. In other words, the rows of the matrix $\sigma(v)$ are not necessarily linearly independent, although they may be, as we show in the following example.

Example 1. Let $R$ be a finite commutative Frobenius ring and let $G=\left\{g_{1}, g_{2}, \ldots, g_{n}\right\}$ be a group. Let $v_{1}=\sum 0 g_{i}$. Then $\sigma\left(v_{1}\right)$ is the all zero matrix and $C\left(v_{1}\right)=\{\mathbf{0}\}$. Let $v_{2}=\sum \alpha_{i} g_{i}$ with $\alpha_{j}=1$ for some $j$ and $\alpha_{i}=0$ for $i \neq j$. Then $\sigma(v)$ is permutation equivalent to $I_{n}$, the $n$ by $n$ identity matrix, which gives that $C\left(v_{2}\right)=R^{n}$.

We will say that two matrices are equivalent if they generate equivalent codes.

Example 2. Let $v=\left(1+s+s^{2}+s^{3}\right)(1+t) \in \mathbb{F}_{2} M_{16}$ where $M_{16}=\langle s, t| s^{8}=t^{2}=1$, st $\left.=t s^{5}\right\rangle$ is the modular group of order 16 . Then,

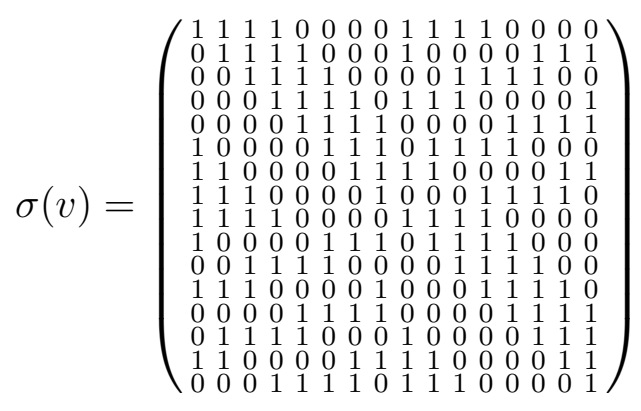

and $\sigma(v)$ is equivalent to

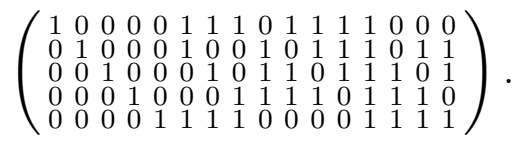


Clearly, $C(v)$ is the $[16,5,8]$ Reed-Muller code.

We shall now show that the codes we construct are actually ideals in the group ring. We use this to get information about the automorphism group of the constructed code.

Theorem 2.1. Let $R$ be a finite commutative Frobenius ring and $G$ a finite group of order $n$. Let $v \in R G$ and let $C(v)$ be the corresponding code in $R^{n}$. Let $I(v)$ be the set of elements of $R G$ such that $\sum \alpha_{i} g_{i} \in I(v)$ if and only if $\left(\alpha_{1}, \alpha_{2}, \ldots, \alpha_{n}\right) \in C(v)$. Then $I(v)$ is a left ideal in $R G$.

Proof. The rows of $\sigma(v)$ consist precisely of the vectors that correspond to the elements $h v$ in $R G$ where $h$ is any element of $G$. The sum of any two elements in $I(v)$ corresponds exactly to the sum of the corresponding elements in $C(v)$ and so $I(v)$ is closed under addition.

Let $w_{1}=\sum \beta_{i} g_{i} \in R G$. Then if $w_{2}$ corresponds to a vector in $C(v)$, it is of the form $\sum \gamma_{j} h_{j} v$. Then $w_{1} w_{2}=\sum \beta_{i} g_{i} \sum \gamma_{i} h_{i} v=\sum \beta_{i} \gamma_{j} g_{i} h_{j} v$ which corresponds to an element in $C(v)$ and gives that the element is in $I(v)$. Therefore $I(v)$ is a left ideal of $R G$.

Example 3. Let $v=1+b a+b a^{2}+b a^{3} \in \mathbb{F}_{2} D_{8}$ where $\langle a, b\rangle \cong D_{8}$. Then $\sigma(v)=$

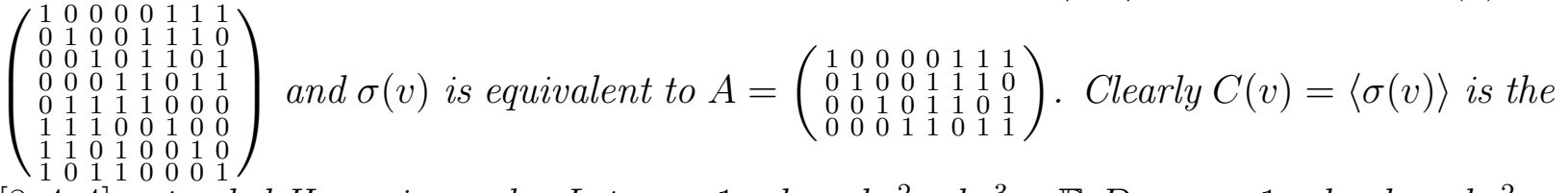
$[8,4,4]$ extended Hamming code. Let $v_{1}=1+b a+b a^{2}+b a^{3} \in \mathbb{F}_{2} D_{8}, v_{2}=1+b+b a+b a^{2} \in$ $\mathbb{F}_{2} D_{8}, v_{3}=1+b+b a+b a^{3} \in \mathbb{F}_{2} D_{8}$ and $v_{4}=1+b+b a^{2}+b a^{3} \in \mathbb{F}_{2} D_{8}$ where $v_{i}$ are the group ring element corresponding to the rows of $A$. Let $I(v)=\left\{\sum_{i=1}^{4} \alpha_{i} v_{i} \mid \alpha_{i} \in \mathbb{F}_{2}\right\}$. Then $I(v)$ is a left ideal of $\mathbb{F}_{2} D_{8}$ and in particular $I(v)$ is the left principle ideal of $\mathbb{F}_{2} D_{8}$ generated by $v$.

Corollary 2.2. Let $R$ be a finite commutative Frobenius ring and $G$ a finite group of order $n$. Let $v \in R G$ and let $C(v)$ be the corresponding code in $R^{n}$. Then the automorphism group of $C(v)$ has a subgroup isomorphic to $G$.

Proof. Since $I(v)$ is an ideal in $R G$ we have that $I(v)$ is held invariant by the action of the elements of $G$. It follows immediately that the automorphism group of $C(v)$ contains $G$ as a subgroup.

We note that our construction gives a natural generalization of cyclic codes since cyclic codes are ideals in $R C_{n}$ where $C_{n}$ is the cyclic group of order $n$. Cyclic codes are held invariant by the cyclic shift whereas our codes are held invariant by the action of the group $G$ on the coordinates. Moreover, this is the strength of our construction technique. Namely, we can construct a code whose automorphism group must contain a given group. In this sense, when the group used is $G$, we can refer to a code that is an ideal in $R G$ as $G$-codes, where $G$ is replaced by the name of the code when known. Therefore, classically we can say cyclic codes, but we can know say dihedral codes or dicyclic codes. When something applies 
to any group we can still say $G$-codes. It is immediate that a code of length $n$ can only be a $G$-code for some $G$ if it has a subgroup of its automorphism group of order $n$.

Example 4. Let $C$ be the extremal $[48,24,12]$ Pless symmetry code. The automorphism group of this code is PSL $(2,47)$. A computation in GAP [12] shows that the only subgroup of PSL $(2,47)$ of order 48 is $D_{48}$. Hence the only possible construction of this code by our technique must have $G=D_{48}$. This construction is given by McLoughlin in [18]. This gives that the Pless symmetry code is, in fact, a dihedral code.

Combining the results in [2], [3], [4], [20], [21] and [22], we have that the automorphism group of a putative $[72,36,16]$ code must have order $1,2,3,4$, or 5 . See [8] for details on the automorphism group and a detailed description of this putative code. Since it is impossible for a group of order 72 to satisfy these we have the following corollary.

Corollary 2.3. The putative $[72,36,16]$ code cannot be of the form $C(v)$ for any $v \in \mathbb{F}_{2} G$ for any group $G$.

Proof. The result follows immediately from Corollary 2.2 and the previous discussion.

Note that a code whose automorphism group is trivial cannot be constructed by this technique.

\subsection{The Family of Rings $R_{k}$}

In this subsection, we shall describe a family of rings which is useful in producing binary formally self-dual codes via their associated Gray maps.

Define the ring $R_{k}$ as

$$
R_{k}=\mathbb{F}_{2}\left[u_{1}, u_{2}, \ldots, u_{k}\right] /\left\langle u_{i}^{2}, u_{i} u_{j}-u_{j} u_{i}\right\rangle .
$$

These rings are local rings of characteristic 2 with maximal ideal $\mathfrak{m}=\left\langle u_{1}, u_{2}, \ldots, u_{k}\right\rangle$. This maximal ideal is also necessarily the Jacobson radical of the ring, which can be characterized as the intersection of all maximal ideals. The socle, which is the sum of all minimal ideals, for the ring $R_{k}$ is $\operatorname{Soc}\left(R_{k}\right)=\left\langle u_{1} u_{2} \cdots u_{k}\right\rangle=\mathfrak{m}^{\perp}$. We have that $\left|R_{k}\right|=2^{2^{k}}$. The rings $R_{k}$ were described in [9], [10], and [11].

We can describe a Gray map for $R_{k}$. We define $\phi_{1}\left(a+b u_{1}\right)=(b, a+b)$, where $\phi$ maps $R$ to $\mathbb{F}_{2}^{2}$. Then view $R\left[u_{1}, u_{2}, \ldots, u_{s}\right]$ as $R\left[u_{1}, u_{2}, \ldots, u_{s-1}\right]\left[u_{s}\right]$ and define $\phi_{s}\left(a+b u_{s}\right)=(b, a+b)$. Then the map $\phi_{k}$ is map from $R_{k}$ to $\mathbb{F}_{2}^{2^{k}}$.

The following theorem appears in [11].

Theorem 2.4. Let $C$ be a self-dual code over $R_{k}$, then $\phi_{k}(C)$ is a self-dual code in $\mathbb{F}_{2}^{2^{k}}$.

We shall give several examples where we construct self-dual codes over $R_{k}$ using the method in the paper and then use the Gray map to construct a binary self-dual code of longer length. 


\subsection{Codes, Ideals and Orthogonals}

One of the fundamental results about cyclic codes is that the orthogonal of a cyclic code is again a cyclic code. In this subsection, we generalize this results to codes that are ideals in a group ring. That is we show that if $C$ is a $G$-code for some $G$ then its orthogonal $C^{\perp}$ is also a $G$-code.

Let $I$ be an ideal in a group ring $R G$. Define $\mathcal{R}(C)=\{w \mid v w=0, \forall v \in I\}$. It is immediate that $\mathcal{R}(I)$ is an ideal of $R G$.

Let $v=a_{g_{1}} g_{1}+a_{g_{2}} g_{2}+\ldots a_{g_{n}} g_{n} \in R G$ and $C(v)$ be the corresponding code. Let $\Psi: R G \rightarrow R^{n}$ be the canonical map that sends $a_{g_{1}} g_{1}+a_{g_{2}} g_{2}+\ldots a_{g_{n}} g_{n}$ to $\left(a_{g_{1}}, a_{g_{2}}, \ldots, a_{g_{n}}\right)$. Let $I$ be the ideal $\Psi^{-1}(C)$. Let $\mathbf{w}=\left(w_{1}, w_{2}, \ldots, w_{n}\right) \in C^{\perp}$. Then

$$
\left[\left(a_{g_{j}^{-1} g_{1}}, a_{g_{j}^{-1} g_{2}}, \ldots, a_{g_{j}^{-1} g_{n}}\right),\left(w_{1}, w_{2}, \ldots, w_{n}\right)\right]=0, \forall j .
$$

This gives that

$$
\sum_{i=1}^{n} a_{g_{j}^{-1} g_{i}} w_{i}=0, \forall j
$$

Let $w=\Psi^{-1}(\mathbf{w})=\sum w_{g_{i}} g_{i}$ and define $\overline{\mathbf{w}} \in R G$ to be $\overline{\mathbf{w}}=b_{g_{1}} g_{1}+b_{g_{2}} g_{2}+\cdots+b_{g_{n}} g_{n}$ where

$$
b_{g_{i}}=w_{g_{i}^{-1}} .
$$

Then

$$
\sum_{i=1}^{n} a_{g_{j}^{-1} g_{i}} w_{i}=0 \Longrightarrow \sum_{i=1}^{n} a_{g_{j}^{-1} g_{i}} b_{g_{i}^{-1}}=0 .
$$

Then $g_{j}^{-1} g_{i} g_{i}^{-1}=g_{j}^{-1}$, hence this is the coefficient of $g_{j}^{-1}$ in the product of $\overline{\mathbf{w}}$ and $g_{j}^{-1} v$. This gives that $\overline{\mathbf{w}} \in \mathcal{R}(I)$ if and only if $\mathbf{w} \in C^{\perp}$.

Let $\phi: R^{n} \rightarrow R G$ by $\phi(\mathbf{w})=\overline{\mathbf{w}}$. It is clear that $\phi$ is a bijection between $C^{\perp}$ and $\mathcal{R}\left(\Psi^{-1}(C)\right)$.

Theorem 2.5. Let $C=C(v)$ be a code in $R G$ formed from the vector $v \in R G$. Then $\Psi^{-1}\left(C^{\perp}\right)$ is an ideal of $R G$.

Proof. We have that $\Psi\left(\phi\left(C^{\perp}\right)\right)$ is permutation equivalent to $C^{\perp}$ and $\phi\left(C^{\perp}\right)$ is an ideal and so $\Psi^{-1}(C)$ is an ideal as well.

This is a generalization of the well known result that the dual of a cyclic code is a cyclic code. The action induces by $\overline{\mathbf{w}}$ is the similar to the polynomial $\overline{f(x)}$ used in cyclic codes to generate the ideal in $R[x] /\left\langle x^{n}-1\right\rangle$ corresponding to the dual code.

We can now generalize another important technique used in the theory of cyclic codes. Let $R$ be a finite commutative Frobenius ring that is isomorphic via the Chinese Remainder Theorem to $R_{1} \times R_{2} \times \ldots R_{s}$. Let $C R T: R_{1} \times R_{2} \times \ldots R_{s} \rightarrow R$ be the map induced my the Chinese Remainder Theorem. See [5] for a complete description of the use of this map in coding theory. 
Theorem 2.6. Let $C_{i}$ be a $G$-code over the ring $R_{i}$, that is $C_{i}$ is an ideal in $R_{i} G$. Then $C R T\left(C_{1}, C_{2}, \ldots, C_{s}\right)$ is a $G$-code over $R$.

Proof. Let $g \in G$ and $\mathbf{v}_{i} \in C_{i}$. Then $g \mathbf{v}_{i} \in C_{i}$ for all $i$. Then if $\mathbf{v}=C R T\left(\mathbf{v}_{1}, \mathbf{v}_{2}, \ldots, \mathbf{v}_{s}\right)$ then $g \mathbf{v}=C R T\left(g \mathbf{v}_{1}, g \mathbf{v}_{2}, \ldots, g \mathbf{v}_{s}\right)$ and so $g \mathbf{v} \in C$ giving that $C$ is an ideal in $R G$.

It is well known that any Frobenius ring is isomorphic to the direct product under the Chinese Remainder Theorem of Frobenius local rings. Additionally, a principal ideal ring isomorphic to the direct product under the Chinese Remainder Theorem of chain rings. Therefore, to study $G$-codes what is really necessary is to study $G$-codes over local rings, of which, chain rings are a special family.

\subsection{Self-Orthogonal Codes}

The following is a rephrasing, in more general terms, of Theorem 1 in [13]. Specifically, in [13], $R$ is assumed to be a field. The proof is identical and simply consists of showing that addition and multiplication is preserved.

Theorem 2.7. Let $R$ be a finite commutative Frobenius ring and let $G$ be a group of order $n$. Then the map $\sigma: R G \rightarrow M_{n}(R)$ is an injective ring homomorphism.

For an element $v=\sum \alpha_{i} g_{i} \in R G$, define the element $v^{T} \in R G$ as $v^{T}=\sum \alpha_{i} g_{i}^{-1}$. This is sometimes known as the canonical involution for the group ring. The reason this notation is used in this setting will be apparent by the next lemma.

The following is a straightforward generalization of a result in [13].

Lemma 2.8. Let $R$ be a finite commutative Frobenius ring and let $G$ be a group of order $n$. For an element $v \in R G$, we have that $\sigma(v)^{T}=\sigma\left(v^{T}\right)$.

Proof. The $i j$-th element of $\sigma\left(v^{T}\right)$ is $\alpha_{\left(g_{i}^{-1} g_{j}\right)^{-1}}=\alpha_{g_{j}^{-1} g_{i}}$ which is the $j i$-th element of $\sigma(v)$.

We next give our first result about the structure of our constructed codes.

Lemma 2.9. Let $R$ be a finite commutative Frobenius ring and let $G$ be a group of order $n$. If $v=v^{T}$ and $v^{2}=0$ then $C_{v}$ is a self-orthogonal code.

Proof. If $v=v^{T}$ then $\sigma(v)^{T}=\sigma\left(v^{T}\right)$ by Lemma 2.8. Then we have that $(\sigma(v) \sigma(v))_{i j}$ is the inner-product of the $i$-th and $j$-th rows of $\sigma(v)$. Since $v^{2}=0$, by Theorem 2.7 we have that $\sigma(v) \sigma(v)=\mathbf{0}$. This gives that any two rows of $\sigma(v)$ are orthogonal and hence they generate a self-orthogonal code.

We can now use this lemma to construct self-dual codes. For codes over fields we could simply use the dimension of $\sigma(v)$, however over an arbitrary Frobenius ring we cannot determine the size of the generated code simply from the rank of the matrix. Therefore, we have the following theorem. 
Theorem 2.10. Let $R$ be a finite commutative Frobenius ring and $G$ be a group of order $n$, with $v$ an element in $R G$. If $v=v^{T}, v^{2}=0$ and $\left|C_{v}\right|=|R|^{\frac{n}{2}}$ then $C_{v}$ is a self-dual code.

Proof. By Lemma 2.9 the code $C_{v}$ is self-orthogonal and since $\left|C_{v}\right|=|R|^{\frac{n}{2}}$ we have that $C_{v}$ is self-dual.

Notice that unlike the field case we are not assuming that $n$ is even. For example, let $R=R_{k}$ and $G$ be the trivial group of size 1 with $v=u_{i} e_{G}$ where $e_{G}$ is the identity of the group. Then $\sigma(v)=\left(u_{i}\right)$ and $C_{v}$ is a self-dual code of length 1 .

In the following example, we show the strength of this construction by constructing a code over $R_{1}$ using the alternating group on 4 letters which has an image under the associated Gray map of the length 24 extended Golay code.

Example 5. We shall use the previous results to construct the binary Golay code from the ring $R_{1}$. Let $v=u\left(b+a b+a c+b c^{2}\right)+\left(b c+b c^{2}\right)+(1+u)\left(c^{2}+a b c^{2}\right) \in R_{1} A_{4}$. Then, $C_{v}$ is a self-dual code of length 12 over $R_{1}$. Hence $\phi_{k}(C)$ is a binary self-dual code of length 12 by Theorem 2.4. The binary code $\phi_{k}(C)$ has a generator matrix of the following form:

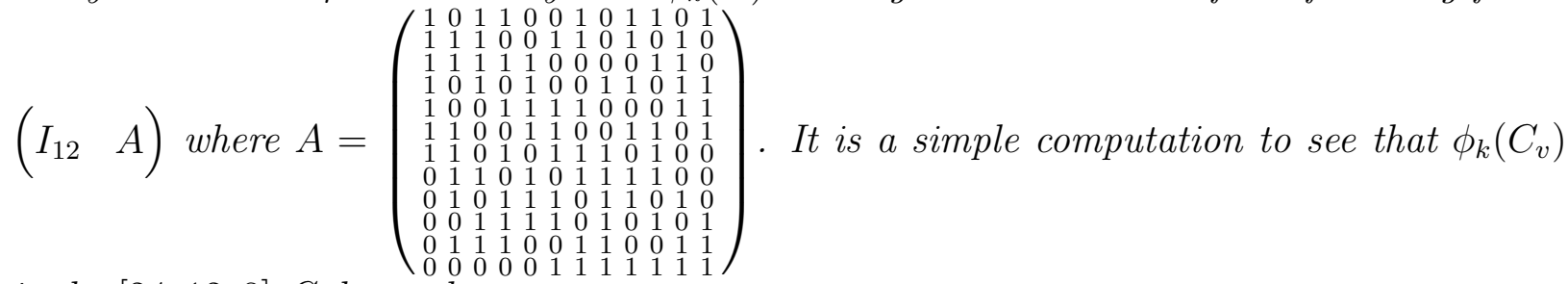
is the $[24,12,8]$ Golay code.

Lemma 2.11. Let $R$ be a finite commutative Frobenius ring and let $G$ be a group of order $n$. If $v=\sum \alpha_{i} g_{i}$ and $w=\alpha_{i} g_{i}$ h for some $h \in G$ then $C_{v}$ and $C_{w}$ are equivalent codes.

Proof. The generator matrix for $C_{w}$ is formed from the generator matrix of $C_{v}$ by permuting the columns corresponding to multiplication of the elements of $G$ by $h$. Hence, the codes are equivalent.

Example 6. Let $v_{1}=1+x z+y z+x y z \in \mathbb{F}_{2}\left(C_{2} \times C_{2} \times C_{2}\right)$ where $\langle x, y, z\rangle \cong C_{2} \times C_{2} \times C_{2}$. Now $\sigma\left(v_{1}\right)$ is equivalent to $\left(\begin{array}{llllllll}1 & 0 & 0 & 0 & 0 & 1 & 1 & 1 \\ 0 & 1 & 0 & 0 & 1 & 0 & 1 & 1 \\ 0 & 0 & 1 & 0 & 1 & 1 & 0 & 1 \\ 0 & 0 & 0 & 1 & 1 & 1 & 1 & 1 \\ 0\end{array}\right)$. The code $C\left(v_{1}\right)$ is the the $[8,4,4]$ extended Hamming code. Next, let us consider $v_{2}=(1+x z+y z+x y z) y=y+x z+z+x y z \in \mathbb{F}_{2}\left(C_{2} \times C_{2} \times C_{2}\right)$. Then $\sigma\left(v_{2}\right)$ is equivalent to $\left(\begin{array}{llllllll}1 & 0 & 0 & 1 & 0 & 0 & 1 & 1 \\ 0 & 1 & 0 & 1 & 0 & 1 & 0 & 1 \\ 0 & 0 & 1 & 0 & 1 & 1 & 0 \\ 0 & 0 & 0 & 0 & 1 & 1 & 1 & 1\end{array}\right)$. Clearly $C\left(v_{1}\right)$ is equivalent to $C\left(v_{2}\right)$.

\section{Binary Golay Code}

The self-dual binary Golay code is one of the most interesting codes. It has interesting connections to the Leech lattice and is an extension of the length 23 perfect Golay code. We 
shall now consider constructions of the $[24,12,8]$ binary Golay code from $\mathbb{F}_{2} G$ for various groups $G$.

It is well known that the automorphism group of the $[24,12,8]$ code is the Mathieu group $M_{24}$. Therefore, the only possible groups that can work for our construction are

$$
S L(2,3), S_{4}, D_{24},\left(C_{6} \times C_{2}\right) \rtimes C_{2}, C_{3} \times D_{8}, C_{2} \times A_{4} \text { and } C_{2}^{2} \times D_{6} \cdot{ }^{1}
$$

Initially, it was shown in [1] that the $[24,12,8]$ could be constructed from ideals in the group algebra $\mathbb{F}_{2} S_{4}$ where $S_{4}$ is the symmetric group on 4 elements. See also [17] for similar results. In [19], the $[24,12,8]$ code was constructed from $\mathbb{F}_{2} D_{24}$. We shall now separately consider the remaining cases.

\subsection{The Group $C_{3} \times D_{8}$}

We begin by considering the group $C_{3} \times D_{8}$. Let $v$ be the element

$$
v=\sum_{i=1}^{4}\left[a^{i-1}\left(\alpha_{i}+\alpha_{i+4} z+\alpha_{i+8} z^{2}\right)+b a^{i-1}\left(\alpha_{i+12}+\alpha_{i+16} z+\alpha_{i+20} z^{2}\right)\right] \in \mathbb{F}_{2}\left(C_{3} \times D_{8}\right)
$$

where $\langle z\rangle=C_{3},\langle a, b\rangle=D_{8}$ and $\alpha_{i} \in \mathbb{F}_{2}$. Now

$$
\sigma(v)=\left(\begin{array}{cc}
A & B \\
B & A
\end{array}\right)
$$

where $A=\left(\begin{array}{ccc}A_{1} & A_{2} & A_{3} \\ A_{3} & A_{1} & A_{2} \\ A_{2} & A_{3} & A_{1}\end{array}\right), B=\left(\begin{array}{ccc}B_{1} & B_{2} & B_{3} \\ B_{3} & B_{1} & B_{2} \\ B_{2} & B_{3} & B_{1}\end{array}\right)$

$$
\begin{aligned}
& A_{1}=\operatorname{cir}\left(\alpha_{1}, \alpha_{2}, \alpha_{3}, \alpha_{4}\right), \\
& A_{2}=\operatorname{cir}\left(\alpha_{5}, \alpha_{6}, \alpha_{7}, \alpha_{8}\right), \\
& A_{3}=\operatorname{cir}\left(\alpha_{9}, \alpha_{10}, \alpha_{11}, \alpha_{12}\right), \\
& B_{1}=\operatorname{riv}\left(\alpha_{13}, \alpha_{14}, \alpha_{15}, \alpha_{16}\right), \\
& B_{2}=\operatorname{rir}\left(\alpha_{17}, \alpha_{18}, \alpha_{19}, \alpha_{20}\right), \\
& B_{3}=\operatorname{rir}\left(\alpha_{21}, \alpha_{22}, \alpha_{23}, \alpha_{24}\right)
\end{aligned}
$$

and $\operatorname{cir}\left(\alpha_{1}, \alpha_{2}, \ldots, \alpha_{n}\right), \operatorname{rcir}\left(\alpha_{1}, \alpha_{2}, \ldots, \alpha_{n}\right)$ are circulant and reverse circulant matrices respectively and $\alpha_{1}, \alpha_{2}, \ldots, \alpha_{n}$ is the first row of the respective matrices. Clearly $\langle\sigma(v)\rangle$ is self-dual if $\sigma(v)^{T}=\sigma(v)$. Now, $\sigma(v)^{T}=\sigma(v)$ if and only if $a_{2}=a_{4}, a_{5}=a_{9}, a_{6}=a_{12}$,

\footnotetext{
${ }^{1}$ These groups are SmallGroup $(24, i)$ for $i \in\{3,6,8,10,12,13,14\}$ according to the GAP system [12].
} 
$a_{7}=a_{11}, a_{8}=a_{10}, a_{17}=a_{21}, a_{18}=a_{22}, a_{19}=a_{23}$ and $a_{20}=a_{24}$. Next, consider elements of $\mathbb{F}_{2}\left(C_{3} \times D_{8}\right)$ of the form

$$
\begin{aligned}
& \left\{\alpha_{1}+\alpha_{2}\left(a+a^{3}\right)+\alpha_{3} a^{2}+\alpha_{4}\left(z+z^{2}\right)+\alpha_{5} a z\left(1+a^{2} z\right)+\alpha_{6} a^{2} z(1+z)+\alpha_{7} a z\left(a^{2}+z\right)\right. \\
& \left.\quad+\sum_{i=1}^{4} b\left(\alpha_{i+7}+\alpha_{i+11}\left(z+z^{2}\right)\right) a^{i-1} \mid \alpha_{i} \in \mathbb{F}_{2}\right\}
\end{aligned}
$$

and in particular the element $v_{1}=1+b[(\hat{a}+1)+(1+a)(\hat{z}+1)]$ of this set where $\hat{a}=\sum_{i=0}^{3} a^{i}$ and $\hat{z}=\sum_{i=0}^{2} z^{i}$. The matrix $\sigma\left(v_{1}\right)$ is equivalent to

$$
\left(\begin{array}{ll}
I & A \\
A & I
\end{array}\right)
$$

where

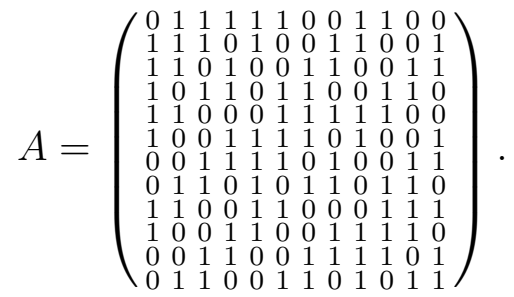

It is a small computation to see that $C\left(v_{1}\right)$ is the $[24,12,8]$ code. Moreover, it can be shown that the above set contains 128 elements that generate the $[24,12,8]$ code.

\subsection{The Group $C_{2} \times A_{4}$}

Next we consider the group $C_{2} \times A_{4}$. Let $v$ be the element

$$
\begin{aligned}
v & =\sum_{i=1}^{3}\left(\alpha_{4 i-3}+\alpha_{4 i-2} a+\alpha_{4 i-1} b+\alpha_{4 i} a b+\alpha_{4 i+9} x+\alpha_{4 i+10} x a+\alpha_{4 i+11} x b+\alpha_{4 i+21} x a b\right) c^{i-1} \\
& \in \mathbb{F}_{2}\left(C_{2} \times A_{4}\right)
\end{aligned}
$$

where $\langle x\rangle=C_{2}, a=(1,2)(3,4), b=(1,3)(2,4)$ and $c=(1,2,3)$ and $\alpha_{i} \in \mathbb{F}_{2}$. Now

$$
\sigma(v)=\left(\begin{array}{ll}
A & B \\
B & A
\end{array}\right)
$$

where $A=\left(\begin{array}{ccc}A_{2} & A_{2} & A_{3} \\ A_{4} & A_{5} & A_{6} \\ A_{7} & A_{8} & A_{9}\end{array}\right), B=\left(\begin{array}{ccc}B_{2} & B_{2} & B_{3} \\ B_{4} & B_{5} & B_{6} \\ B_{7} & B_{8} & B_{9}\end{array}\right)$,

$A_{1}=b c\left(\alpha_{1}, \alpha_{2}, \alpha_{3}, \alpha_{4}\right), A_{2}=b c\left(\alpha_{5}, \alpha_{6}, \alpha_{7}, \alpha_{8}\right), A_{3}=b c\left(\alpha_{9}, \alpha_{10}, \alpha_{11}, \alpha_{12}\right)$,

$A_{4}=b c\left(\alpha_{9}, \alpha_{12}, \alpha_{10}, \alpha_{11}\right), A_{5}=b c\left(\alpha_{1}, \alpha_{4}, \alpha_{2}, \alpha_{3}\right), A_{6}=b c\left(\alpha_{5}, \alpha_{8}, \alpha_{6}, \alpha_{7}\right)$,

$A_{7}=b c\left(\alpha_{5}, \alpha_{7}, \alpha_{8}, \alpha_{6}\right), A_{8}=b c\left(\alpha_{9}, \alpha_{11}, \alpha_{12}, \alpha_{10}\right), A_{9}=b c\left(\alpha_{1}, \alpha_{3}, \alpha_{4}, \alpha_{2}\right)$, 
$B_{1}=b c\left(\alpha_{13}, \alpha_{14}, \alpha_{15}, \alpha_{16}\right), B_{2}=b c\left(\alpha_{17}, \alpha_{18}, \alpha_{19}, \alpha_{20}\right), B_{3}=b c\left(\alpha_{21}, \alpha_{22}, \alpha_{23}, \alpha_{24}\right)$,

$B_{4}=b c\left(\alpha_{21}, \alpha_{24}, \alpha_{22}, \alpha_{23}\right), B_{5}=b c\left(\alpha_{13}, \alpha_{16}, \alpha_{14}, \alpha_{15}\right), B_{6}=b c\left(\alpha_{17}, \alpha_{20}, \alpha_{18}, \alpha_{19}\right)$,

$B_{7}=b c\left(\alpha_{17}, \alpha_{19}, \alpha_{20}, \alpha_{18}\right), B_{8}=b c\left(\alpha_{21}, \alpha_{23}, \alpha_{24}, \alpha_{22}\right)$ and $B_{9}=b c\left(\alpha_{13}, \alpha_{15}, \alpha_{16}, \alpha_{14}\right)$

where $b c(a, b, c, d)$ is a matrix that takes the form $\left(\begin{array}{llll}a & b & c & d \\ b & a & d & c \\ c & d & a & b \\ d & c & b & a\end{array}\right)$. Now, $\sigma(v)=\sigma(v)^{T}$ if and only if $a_{5}=a_{9}, a_{6}=a_{12}, a_{7}=a_{10}, a_{8}=a_{11}, a_{17}=a_{21}, a_{18}=a_{24}, a_{19}=a_{24}$ and $a_{20}=a_{23}$. Next, consider elements of $\mathbb{F}_{2}\left(C_{2} \times A_{4}\right)$ of the form

$$
\begin{aligned}
& \left\{\sum _ { i = 0 } ^ { 1 } x ^ { i } \left(\left(\alpha_{8 i+1}+\alpha_{8 i+2} a+\alpha_{8 i+3} b+\alpha_{8 i+4} a b\right)+\right.\right. \\
& \left.\left.\quad\left(\alpha_{8 i+5}+\alpha_{8 i+6} a+\alpha_{8 i+7} b+\alpha_{8 i+8} a b\right)\left(c+c^{2}\right)\right) \mid \alpha_{i} \in \mathbb{F}_{2}\right\},
\end{aligned}
$$

and in particular the element $v_{1}=1+x\left(1+b(1+a)\left(1+c^{2}\right)\right)+x a(1+b) c$ of this set. The matrix $\sigma\left(v_{1}\right)$ is equivalent to

$$
\left(\begin{array}{ll}
I & A \\
A & I
\end{array}\right)
$$

where

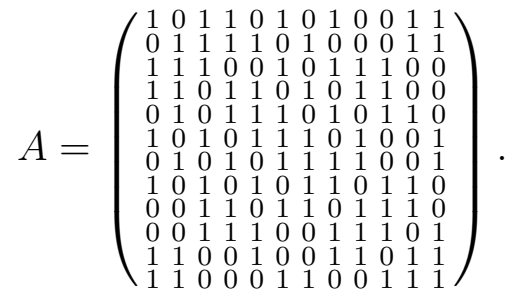

It is a small computation to see that $C\left(v_{1}\right)$ is the $[24,12,8]$ code. Moreover, it can be shown that the above set contains 384 elements that generate the $[24,12,8]$ code.

\subsection{The Group $G=\left(C_{6} \times C_{2}\right) \rtimes C_{2}$}

Next we consider the group $G=\left(C_{6} \times C_{2}\right) \rtimes C_{2}$. Let $v$ be the element

$$
\begin{aligned}
v & =\sum_{i=1}^{4}\left(\alpha_{i} y^{i-1}+\alpha_{i+4} x y^{i-1}+\alpha_{i+8} x^{2} y^{i-1}+\alpha_{i+12} y^{i-1} z+\alpha_{i+16} x y^{i-1} z+\alpha_{i+20} x^{2} y^{i-1} z\right) \\
& \in \mathbb{F}_{2}\left(\left(C_{6} \times C_{2}\right) \rtimes C_{2}\right)
\end{aligned}
$$


where $\left(C_{6} \times C_{2}\right) \rtimes C_{2}=\left\langle x, y, z \mid x^{3}=y^{4}=z^{2}=1, x y=y x^{2}, x z=z x, y z=z y^{3}\right\rangle$ and $\alpha_{i} \in \mathbb{F}_{2}$. Now,

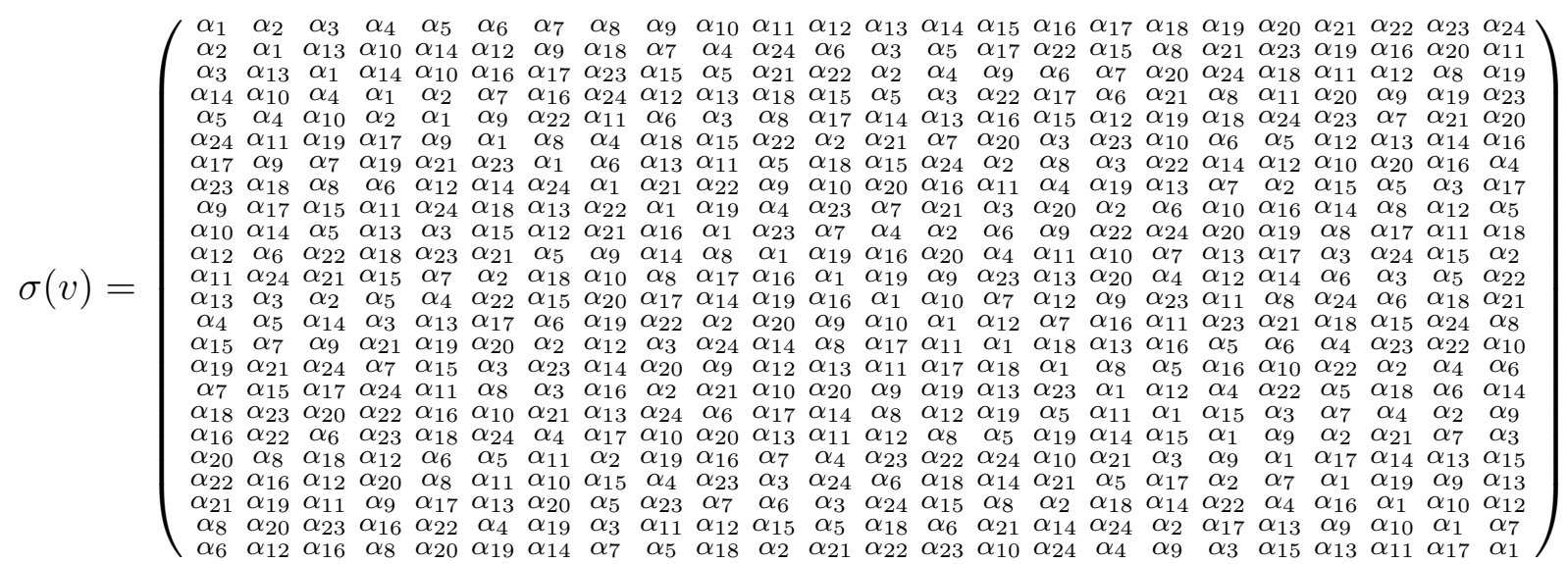

and $\sigma(v)=\sigma(v)^{T}$ if and only if $a_{4}=a_{14}, a_{6}=a_{24}, a_{7}=a_{17}, a_{8}=a_{23}, a_{11}=a_{12}, a_{16}=a_{19}$ and $a_{21}=a_{22}$. Next, consider elements of $\mathbb{F}_{2}\left(\left(C_{6} \times C_{2}\right) \rtimes C_{2}\right)$ of the form

$$
\begin{aligned}
& \left\{\sum_{i=1}^{4}\left(\alpha_{i} y^{i-1}+\alpha_{i+4} x y^{i-1}\right)+\sum_{i=1}^{2}\left(\alpha_{i+8} x^{2} y^{i-1}+\alpha_{i+12} y^{i+1} z\right)+\left(\alpha_{11} x^{2} y^{2}+\alpha_{17} x^{2} z\right)(1+y)\right. \\
& \left.\quad+\alpha_{4} y z+\alpha_{6} x^{2} y^{3} z+\alpha_{7} x z+x^{2} y^{2} z \alpha_{8}+\alpha_{12} z+\alpha_{14} x y^{2} z+\alpha_{15} x y z+\alpha_{16} x y^{3} z\right\}
\end{aligned}
$$

and in particular the element $v_{1}=1+\left[a+b+b^{3}+\left(a+a^{2}\right)\left(b^{2}+b^{3}\right)\right] c$ of this set. The matrix $\sigma\left(v_{1}\right)$ is equivalent to

$$
\left(\begin{array}{ll}
I & A
\end{array}\right)
$$

where

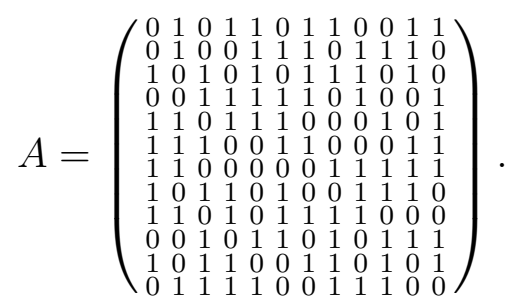

It is a small computation to see that $C\left(v_{1}\right)$ is the $[24,12,8]$ code. Moreover, it can be shown that the above set contains 576 elements that generate the $[24,12,8]$ code.

\subsection{The Group $S L(2,3)$}

Next we consider the group $S L(2,3)$. Let $v$ be the element

$$
v=\sum_{i=1}^{6} x^{i-1}\left(\alpha_{i}+\alpha_{6+i} y+\alpha_{12+i} y^{2}+\alpha_{18+i} y^{2} x\right) \in \mathbb{F}_{2} S L(2,3)
$$


where $S L(2,3)=\left\langle x, y \mid x^{3}=y^{3}=(x y)^{2}\right\rangle$ and $\alpha_{i} \in \mathbb{F}_{2}$. Now,

$$
\sigma(v)=\left(\begin{array}{cccc}
A_{1} & A_{2} & A_{3} & A_{4} \\
A_{5} & A_{6} & A_{7} & A_{8} \\
A_{9} & A_{10} & A_{11} & A_{12} \\
A_{13} & A_{14} & A_{15} & A_{16}
\end{array}\right)
$$

where $A_{1}=\operatorname{circ}\left(\alpha_{1}, \alpha_{2}, \alpha_{3}, \alpha_{4}, \alpha_{5}, \alpha_{6}\right), A_{2}=\operatorname{circ}\left(\alpha_{7}, \alpha_{8}, \alpha_{9}, \alpha_{10}, \alpha_{11}, \alpha_{12}\right)$, $A_{3}=\operatorname{circ}\left(\alpha_{13}, \alpha_{14}, \alpha_{15}, \alpha_{16}, \alpha_{17}, \alpha_{18}\right), A_{4}=\operatorname{circ}\left(\alpha_{19}, \alpha_{20}, \alpha_{21}, \alpha_{22}, \alpha_{23}, \alpha_{24}\right)$, $A_{5}=\operatorname{circ}\left(\alpha_{16}, \alpha_{22}, \alpha_{8}, \alpha_{13}, \alpha_{19}, \alpha_{11}\right), A_{6}=\operatorname{circ}\left(\alpha_{1}, \alpha_{21}, \alpha_{14}, \alpha_{4}, \alpha_{24}, \alpha_{17}\right)$, $A_{7}=\operatorname{circ}\left(\alpha_{7}, \alpha_{20}, \alpha_{5}, \alpha_{10}, \alpha_{23}, \alpha_{2}\right), A_{8}=\operatorname{circ}\left(\alpha_{18}, \alpha_{12}, \alpha_{6}, \alpha_{15}, \alpha_{9}, \alpha_{3}\right)$, $A_{9}=\operatorname{circ}\left(\alpha_{10}, \alpha_{15}, \alpha_{21}, \alpha_{7}, \alpha_{18}, \alpha_{24}\right), A_{10}=\operatorname{circ}\left(\alpha_{16}, \alpha_{6}, \alpha_{20}, \alpha_{13}, \alpha_{3}, \alpha_{23}\right)$, $A_{11}=\operatorname{circ}\left(\alpha_{1}, \alpha_{12}, \alpha_{19}, \alpha_{4}, \alpha_{9}, \alpha_{22}\right), A_{12}=\operatorname{circ}\left(\alpha_{2}, \alpha_{17}, \alpha_{11}, \alpha_{5}, \alpha_{14}, \alpha_{8}\right)$, $A_{13}=\operatorname{circ}\left(\alpha_{9}, \alpha_{14}, \alpha_{20}, \alpha_{12}, \alpha_{17}, \alpha_{23}\right), A_{14}=\operatorname{circ}\left(\alpha_{15}, \alpha_{5}, \alpha_{19}, \alpha_{18}, \alpha_{2}, \alpha_{22}\right)$, $A_{15}=\operatorname{circ}\left(\alpha_{6}, \alpha_{11}, \alpha_{24}, \alpha_{3}, \alpha_{8}, \alpha_{21}\right), A_{16}=\operatorname{circ}\left(\alpha_{1}, \alpha_{16}, \alpha_{10}, \alpha_{4}, \alpha_{13}, \alpha_{7}\right)$.

Now, $\sigma(v)=\sigma(v)^{T}$ if and only if $\alpha_{2}=\alpha_{6}, \alpha_{3}=\alpha_{5}, \alpha_{7}=\alpha_{16}, \alpha_{8}=\alpha_{11}, \alpha_{9}=\alpha_{19}$, $\alpha_{10}=\alpha_{13}, \alpha_{12}=\alpha_{22}, \alpha_{14}=\alpha_{24}, \alpha_{15}=\alpha_{18}, \alpha_{17}=\alpha_{21}$ and $\alpha_{20}=\alpha_{23}$. Next, consider elements of $\mathbb{F}_{2} S L(2,3)$ of the form:

$$
\begin{aligned}
& \left\{\alpha_{1}+\alpha_{2}\left(x+x^{5}\right)+\alpha_{3}\left(x^{2}+x^{4}\right)+\alpha_{4} x^{3}+\alpha_{5}\left(y+x^{3} y^{2}\right)+\alpha_{6}\left(x y+x^{4} y\right)+\alpha_{7}\left(x^{2} y+y^{2} x\right)\right. \\
& \quad+\alpha_{8}\left(x^{3} y+y^{2}\right)+\alpha_{9}\left(x^{5} y+x^{3} y^{2} x\right)+\alpha_{10}\left(x y^{2}+x^{5} y^{2} x\right)+\alpha_{11}\left(x^{2} y^{2}+x^{5} y^{2}\right) \\
& \left.\quad+\alpha_{12}\left(x^{4} y^{2}+x^{2} y^{2} x\right)+\alpha_{13}\left(x y^{2} x+x^{4} y^{2} x\right) \mid \alpha_{i} \in \mathbb{F}_{2}\right\} .
\end{aligned}
$$

It can be shown that it is not possible to construct the $[24,12,8]$ from any element of this set.

\subsection{The Group $C_{2}^{2} \times D_{6}$}

Next we consider the group $C_{2}^{2} \times D_{6}$. Let $v$ be the element

$v=\sum_{i=0}^{2}\left[\left(\alpha_{i+1}+\alpha_{i+4} z+\alpha_{i+7} w+\alpha_{i+10} z w\right)+b\left(\alpha_{i+13}+\alpha_{i+16} z+\alpha_{i+19} w+\alpha_{i+22} z w\right)\right] a^{i} \in \mathbb{F}_{2}\left(C_{2}^{2} \times D_{6}\right)$

where $\langle z, w\rangle=C_{2}^{2},\langle a, b\rangle=D_{6}$ and $\alpha_{i} \in \mathbb{F}_{2}$. Now

$$
\sigma(v)=\left(\begin{array}{ll}
A & B \\
B & A
\end{array}\right)
$$


where $A=\left(\begin{array}{cccc}A_{1} & A_{2} & A_{3} & A_{4} \\ A_{2} & A_{1} & A_{4} & A_{3} \\ A_{3} & A_{4} & A_{1} & A_{2} \\ A_{4} & A_{3} & A_{2} & A_{1}\end{array}\right), B=\left(\begin{array}{cccc}B_{1} & B_{2} & B_{3} & B_{4} \\ B_{2} & B_{1} & B_{4} & B_{3} \\ B_{3} & B_{4} & B_{1} & B_{2} \\ B_{4} & B_{3} & B_{2} & B_{1}\end{array}\right), A_{1}=\operatorname{cir}\left(\alpha_{1}, \alpha_{2}, \alpha_{3}\right), A_{2}=$ $\operatorname{cir}\left(\alpha_{4}, \alpha_{5}, \alpha_{6}\right), A_{3}=\operatorname{cir}\left(\alpha_{7}, \alpha_{8}, \alpha_{9}\right), A_{4}=\operatorname{cir}\left(\alpha_{10}, \alpha_{11}, \alpha_{12}\right), B_{1}=\operatorname{rcir}\left(\alpha_{13}, \alpha_{14}, \alpha_{15}\right), B_{2}=$ $\operatorname{rcir}\left(\alpha_{16}, \alpha_{17}, \alpha_{18}\right), B_{3}=\operatorname{rcir}\left(\alpha_{19}, \alpha_{20}, \alpha_{21}\right)$ and $B_{4}=\operatorname{rcir}\left(\alpha_{22}, \alpha_{23}, \alpha_{24}\right)$.

Now, $\sigma(v)=\sigma(v)^{T}$ if and only if $\alpha_{2}=\alpha_{3}, \alpha_{5}=\alpha_{6}, \alpha_{8}=\alpha_{9}$ and $\alpha_{11}=\alpha_{12}$. Next, consider elements of $\mathbb{F}_{2}\left(C_{2}^{2} \times D_{6}\right)$ of the form

$$
\begin{aligned}
& \left\{\alpha_{1}+\alpha_{3} z+\alpha_{5} w+\alpha_{7} z w+\left(a+a^{2}\right)\left(\alpha_{2}+\alpha_{4} z+\alpha_{6} w+\alpha_{8} z w\right)\right. \\
& \left.+\sum_{i=0}^{2}+b a^{i}\left(\alpha_{i+13}+\alpha_{i+16} z+\alpha_{i+19} w+\alpha_{i+22} z w\right)\right\} .
\end{aligned}
$$

It can be shown that it is not possible to construct the $[24,12,8]$ Golay code from any element of this set.

We summarize these results in the following: The $[24,12,8]$ Type II code can be constructed in $\mathbb{F}_{2} G$ precisely for the following groups of order 24: $S_{4}, D_{24}, C_{3} \times D_{8}, C_{2} \times A_{4}$ and $\left(C_{6} \times C_{2}\right) \rtimes C_{2}$.

\section{The Dihedral Group}

In this section, we shall describe these techniques for generating codes for the dihedral group. Let $D_{2 k}$ be the dihedral group of order $2 k$. We describe the group by $D_{2 k}=\langle a, b| a^{2}=b^{k}=$ $\left.1, a b=b^{-1} a\right\rangle$. The ordering of the elements for the map $\sigma$ is $1, b, b^{2}, \ldots, b^{k-1}, a, a b, a b^{2}, \ldots, a b^{k-1}$. It is this group that McLoughlin used in [18] to give a construction of the binary $[48,24,12]$ extremal Type II code.

Let $v=\sum \alpha_{a^{i}, b^{j}} a^{i} b^{j}$. In this case, the matrix $\sigma(v)$ is of the form:

$$
\left(\begin{array}{cccccccccc}
\alpha_{1} & \alpha_{b} & \alpha_{b^{2}} & \ldots & \alpha_{b^{k-1}} & \alpha_{a} & \alpha_{a b} & \alpha_{a b^{2}} & \ldots & \alpha_{a b^{k-1}} \\
\alpha_{b^{k-1}} & \alpha_{1} & \alpha_{b} & \ldots & \alpha_{b^{k-2}} & \alpha_{a b} & \alpha_{a b^{2}} & \alpha_{a b^{3}} & \ldots & \alpha_{a} \\
\vdots & \vdots & \vdots & \vdots & \vdots & \vdots & \vdots & \vdots & \vdots & \vdots \\
\alpha_{b} & \alpha_{b^{2}} & \alpha_{b^{3}} & \ldots & \alpha_{1} & \alpha_{a b^{k-1}} & \alpha_{a} & \alpha_{a b} & \ldots & \alpha_{a b^{k-2}} \\
\alpha_{a} & \alpha_{a b} & \alpha_{a b^{2}} & \ldots & \alpha_{a b^{k-1}} & \alpha_{1} & \alpha_{b} & \alpha_{b^{2}} & \ldots & \alpha_{b^{k-1}} \\
\alpha_{a b} & \alpha_{a b^{2}} & \alpha_{a b^{3}} & \ldots & \alpha_{a} & \alpha_{b^{k-1}} & \alpha_{1} & \alpha_{b} & \ldots & \alpha_{b^{k-2}} \\
\vdots & \vdots & \vdots & \vdots & \vdots & \vdots & \vdots & \vdots & \vdots & \vdots \\
\alpha_{a b^{k-1}} & \alpha_{a} & \alpha_{a b} & \ldots & \alpha_{a b^{k-2}} & \alpha_{b} & \alpha_{b^{2}} & \alpha_{b^{3}} & \ldots & \alpha_{1}
\end{array}\right)
$$

This gives that $\sigma(v)$ is of the form:

$$
\left(\begin{array}{ll}
A & B \\
B & A
\end{array}\right)
$$


where $A$ is a circulant matrix and $B$ is a reverse circulant matrix.

We begin by proving a lemma.

Lemma 4.1. Let $R$ be a finite commutative Frobenius ring of characteristic 2. Let $C$ be the code generated by a matrix $M$ of the form

$$
\left(\begin{array}{cc}
I_{k} & B \\
B & I_{k}
\end{array}\right),
$$

where $B$ is a symmetric $k$ by $k$ matrix. If the free rank of $C$ is $k$ then $C$ is self-dual.

Proof. Let $D=\left\langle\left(I_{k} \mid B\right)\right\rangle$ and $D^{\prime}=\left\langle\left(B \mid I_{k}\right)\right\rangle$. The inner-product of the $i$-th row of $\left(I_{k} \mid B\right)$ and the $j$-th row of $\left(B \mid I_{k}\right)$ is $B_{i, j}+B_{j, i}=0$ since $B_{i, j}=B_{j, i}$ and the characteristic is 2 . Therefore $D^{\prime}=D^{\perp}$ since $\left|D \| D^{\prime}\right|=|R|^{n}$.

The code $C=\left\langle D, D^{\perp}\right\rangle$. If $D \neq D^{\perp}$ then $|C|>|D|$. However, we are assuming that the free rank of $C$ is $k$. Hence $C=D=D^{\perp}$. This gives that $C$ is a self-dual code.

In [13], Hurley proves that $C_{v}$ is self-dual over $\mathbb{F}_{2}$ if $v \in \mathbb{F}_{2} D_{24}, v^{2}=0$ and the dimension is $\frac{n}{2}$. We can expand this by showing the following which eliminates the need for $v$ to satisfy $v^{2}=0$.

Theorem 4.2. Let $R$ be a finite commutative Frobenius ring of characteristic 2 and let $v \in R D_{n}$ with $v=\sum \alpha_{i} h_{i}$ where only one $\alpha_{a^{0} b^{i}}$ is 1 and the rest are 0 . If $C_{v}$ has free rank $k$, then $C_{v}$ is a self-dual code.

Proof. Since only one $\alpha_{2 i}$ is 1 and the rest are 0 , the generator matrix of $C_{v}$ is permutation equivalent to a matrix of the form:

$$
\left(\begin{array}{cc}
I_{k} & B \\
B & I_{k}
\end{array}\right)
$$

where $B$ is a reverse circulant matrix and hence symmetric. Then, by Lemma 4.1, we have the result.

To show the importance of the strengthening of this result, consider the element $v=$ $1+a b \in \mathbb{F}_{2} D_{2 k}$ where $k$ is greater than 2 . Then $\left(1 e_{D_{2 k}}+a b\right)^{2} \neq 0$ but $C_{v}$ is a self-dual code. We continue with a larger example.

Example 7. Consider $v \in \mathbb{F}_{2} D_{48}$ such that $\operatorname{dim}\left(C_{v}\right)=24$ and the minimum distance of $C_{v}$ is 10. There are 192 elements $v$ which produce equivalent self-dual codes using the technique. For more information about the importance of this result, see [8].

A common technique for producing self-dual codes is to generate a code with the matrix $\left(I_{\frac{n}{2}} \mid A\right)$ where $A$ is a reverse circulant matrix. Given a code $C$ generated by this matrix we have that $C^{\perp}$ is generated by $\left(A^{T} \mid I_{\frac{n}{2}}\right)$ which is equal to $\left(A \mid I_{\frac{n}{2}}\right)$ since $A$ is symmetric. 
If $C$ is a self-dual code then $\left\langle\left(A \mid I_{\frac{n}{2}}\right)\right\rangle \subseteq\left\langle\left(I_{\frac{n}{2}} \mid A\right)\right\rangle$. This means that the code generated by $\left(\begin{array}{cc}I_{\frac{n}{2}} & A \\ A & I_{\frac{n}{2}}\end{array}\right)$ is the code $C$. Consider the first row of this matrix. Reading this as an element $v \in \mathbb{F}_{2} D_{2 k}$ we have that $C=C(v)$. This gives the following.

Theorem 4.3. Let $C$ be a binary self-dual code generated by $\left(I_{\frac{n}{2}} \mid A\right)$ where $A$ is a reverse circulant matrix then $C=C(v)$ for some $v \in \mathbb{F}_{2} D_{2 k}$.

Applying Corollary 2.3, we have the following.

Corollary 4.4. The putative $[72,36,16]$ Type II code cannot be produced by $\left(I_{\frac{n}{2}} \mid A\right)$ where $A$ is a reverse circulant matrix.

Proof. Corollary 2.3 gives that the $[72,36,16]$ Type II code is not formed from an element in a group algebra and so Theorem 4.3 gives the result.

This corollary eliminates a commonly used technique in the attempt to construct this putative code. Namely, many computational approaches to this problem have been to construct a reverse circulant matrix $A$ and generate the code $\left(I_{\frac{n}{2}} \mid A\right)$. Of course, this technique has not yet produced the code. This corollary give a reason why these attempts have not been successful.

\section{The Cyclic Group Cross the Dihedral Group}

In this section, we shall use the group $G=C_{s} \times D_{2 k}$. Let $C_{s}=\langle h\rangle$ and let $D_{2 k}=\langle a, b| a^{2}=$ $\left.b^{k}=1, a b=b^{-1} a\right\rangle$. We shall order the elements as follows:

$$
\begin{gathered}
\left\{(1,1),(1, b), \ldots,\left(1, b^{k-1}\right),(h, 1),(h, b), \ldots,\left(h, b^{k-1}\right), \ldots,\left(h^{s-1}, 1\right),\right. \\
\left(h^{s-1}, b\right), \ldots,\left(h^{s-1}, b^{k-1}\right),(1, a b), \ldots,\left(1, a b^{k-1}\right),(h, 1),(h, a b), \ldots,\left(h, a b^{k-1}\right), \\
\left.\ldots,\left(h^{s-1}, 1\right),\left(h^{s-1}, a b\right), \ldots,\left(h^{s-1}, a b^{k-1}\right)\right\} .
\end{gathered}
$$

We see that if we choose $v \in R G$ such that only 1 of $\alpha_{\left(h^{i}, a^{0} b^{j}\right)}$ is 1 and the rest are 0 . Then we get a matrix $\sigma(v)$ of the form:

$$
\left(\begin{array}{cc}
I_{k} & B \\
B & I_{k}
\end{array}\right)
$$

where $B$ is of the following form:

$$
B=\left(\begin{array}{ccccc}
1 A & h A & h^{2} A & \ldots & h^{s-1} A \\
h^{s-1} A & 1 A & h A & \ldots & h^{s-2} A \\
\vdots & \vdots & \vdots & \vdots & \vdots \\
h A & h^{2} A & h^{3} A & \ldots & 1 A
\end{array}\right)
$$


where $h^{k} A$ indicates the matrix where the $i, j$-th element is $\left(h^{k}, A_{i, j}\right)$ and $A$ is a reverse circulant matrix.

Theorem 5.1. Let $R$ be a Frobenius ring and let $v \in R C_{s} D_{2 k}$ with $v=\sum \alpha_{i} h_{i}$ where only 1 of $\alpha_{\left(h^{i},\right) a^{0} b^{j}}$ is 1 and the rest are 0 . Let $R$ be a finite commutative Frobenius ring of characteristic 2. If $\left|C_{v}\right|=|R|^{\frac{n}{2}}$, then $C_{v}$ is isodual and hence formally self-dual with respect to any weight enumerator.

Proof. We have that the code $C(v)$ is generated by $\left(I_{k} \mid B\right)$ and then its orthogonal is generated by $\left(B^{T} \mid I_{k}\right)$. Then we have that $B$ is equivalent to $B^{T}$. Therefore $C(v)$ and $C(v)^{\perp}$ are equivalent and therefore, by Lemma 1.1, formally self-dual with respect to any weight enumerator.

Note that if $R$ is a finite field, then the condition in the previous theorem becomes that $\operatorname{dim}\left(C_{v}\right)=\frac{n}{2}$.

Example 8. Let $G$ be the group $C_{3} D_{8}$. There are exactly $2^{12}=4096$ elements in $\mathbb{F}_{2} G$ with the property that $\alpha_{\left(h^{i}, a^{0} b^{j}\right)}$ is equal to 1 when $i=j=0$ and equal to 0 otherwise. Of these 256 have $\operatorname{dim}\left(C_{v}\right)=12$ and 192 of these codes are formally self-dual but not selfdual and 64 are self-dual. Of the 192 formally self-dual codes, 80 have minimum distance 6 which is optimal for Type I codes. As an example, if $v_{1}=1+a\left(b+b(1+b)\left(b h+h^{2}\right)\right)$ then $C_{v_{1}}$ is a formally self-dual code with minimum distance 6 . The remaining 112 formally self-dual codes have have minimum distance 4 and $C_{v_{2}}$ is an example of such a code where $v_{2}=1+a\left(b^{2}+h+b^{3} h+h^{2}+b h^{2}\right)$.

Example 9. Let $G$ be the group $C_{4} D_{8}$ and consider elements of $\mathbb{F}_{2} G$ with the property that $\alpha_{\left(h^{i}, a^{0} b^{j}\right)}$ is equal to 1 when $i=j=0$ and equal to 0 otherwise. Of these elements, there are 2048 that have $\operatorname{dim}\left(C_{v}\right)=16$, of these 512 are self-dual and the remaining 1536 are formally self-dual. Let $v_{1}=1+a(\hat{b}+h) h, v_{2}=1+a\left(b+b^{3}+h+h^{3}+\left(b^{2}+\hat{b}\right) h^{2}+(1+\hat{b}) h^{3}\right)$ and $v_{3}=1+a\left(b(1+h)+\hat{b} h^{2}+(b+\hat{b}) h^{3}\right)$. The code $C_{v_{1}}$ is an example of a formally self-dual with minimum distance 4 , the code $C_{v_{2}}$ is an example of a formally self-dual with minimum distance 6 and the code $C_{v_{3}}$ is an example of a formally self-dual with minimum distance 8. Of the 1536 formally self-dual codes, there are 896 with minimum distance 4, 192 with minimum distance 6 and 448 with minimum distance 8.

Example 10. Let $G$ be $C_{5} D_{8}$ and $v=1+a\left(\left(u+u b+u b^{2}+b^{3}\right)+\left(u+b+b^{2}+u b^{3}\right)(h+\right.$ $\left.\left.h^{4}\right)+\left(1+b+u b^{3}\right)\left(h^{2}+h^{3}\right)\right) \in R_{1} C_{5} D_{8}$. Then $C_{v}=\langle\sigma(v), u \sigma(v)\rangle$ is a self-dual code and its image under $\phi_{1}$ is a binary self-dual [80,40,12] code with an automorphism group of order 160 .

Example 11. Let $G$ be the group $C_{2} D_{26}$ and consider the elements $\mathbb{F}_{2}$ with the properties that $\alpha_{\left(h^{i}, a^{0} b^{j}\right)}$ is equal to 1 when $i=j=0$ and equal to 0 otherwise. Of these elements, there are six inequivalent self-dual $[52,26,10]$ codes. These six elements are as follows: 


\begin{tabular}{|c|c|c|}
\hline$i$ & $v_{i} \in \mathbb{F}_{2}\left(C_{2} D_{26}\right)$ & $\left|A u t\left(C_{v_{i}}\right)\right|$ \\
\hline \hline 1 & $1+a\left(\left(b^{8}+b^{10}+b^{11}+b^{12}\right)+\left(b+b^{2}+b^{3}+b^{4}+b^{5}+b^{6}+b^{8}+b^{9}+b^{11}\right) h\right)$ & 52 \\
\hline 2 & $1+a\left(\left(b^{7}+b^{9}+b^{10}+b^{11}\right)+\left(1+b+b^{2}+b^{3}+b^{5}+b^{7}+b^{8}+b^{10}+b^{11}\right) h\right)$ & 52 \\
\hline 3 & $1+a\left(\left(b^{6}+b^{8}+b^{10}+b^{11}+b^{12}\right)+\left(1+b+b^{2}+b^{3}+b^{5}+b^{7}+b^{8}+b^{11}\right) h\right)$ & 52 \\
\hline 4 & $1+a\left(\left(b^{6}+b^{8}+b^{9}+b^{10}+b^{11}+b^{12}\right)+\left(1+b^{2}+b^{3}+b^{4}+b^{6}+b^{7}+b^{8}\right) h\right)$ & 52 \\
\hline 5 & $1+a\left(\left(b^{5}+b^{8}+b^{9}+b^{10}+b^{12}\right)+\left(b+b^{3}+b^{4}+b^{6}+b^{7}+b^{9}+b^{10}+b^{11}\right) h\right)$ & 52 \\
\hline 6 & $1+a\left(\left(b^{5}+b^{7}+b^{8}+b^{9}+b^{10}+b^{11}+b^{12}\right)+\left(1+b+b^{2}+b^{3}+b^{7}+b^{11}\right) h\right)$ & 52 \\
\hline
\end{tabular}

\section{The Cyclic Case}

In this section, we shall set $G=C_{n}$ the cyclic group of order $n$. Since the inception of cyclic codes, it has been an open question to determine which cyclic codes were self-dual. We shall describe when this occurs.

We focus on the case when $n=2 k$. Let $G=\langle h\rangle$. Then let $h_{i}=h^{i}$. We then use as the ordering of the elements of $G$ :

$$
\left(h_{0}, h_{2}, \ldots, h_{2 k}, h_{1}, h_{3}, \ldots, h_{2 k-1}\right)
$$

That is $g_{i}=h_{2(i-1)}$ for $i=1$ to $k$ and $g_{k+j}=h_{2(j-1)+1}$ for $j=1$ to $k$.

It follows that the form of $\sigma(v)$ is:

$$
\left(\begin{array}{cccccccc}
\alpha_{h_{0}} & \alpha_{h_{2}} & \cdots & \alpha_{h_{2 k}} & \alpha_{h_{1}} & \alpha_{h_{3}} & \cdots & \alpha_{h_{2 k-1}} \\
\alpha_{h_{2 k}} & \alpha_{h_{0}} & \cdots & \alpha_{h_{2 k-2}} & \alpha_{h_{2 k-1}} & \alpha_{h_{1}} & \cdots & \alpha_{h_{2 k-3}} \\
\vdots & \vdots & \ddots & \vdots & \vdots & \vdots & \ddots & \vdots \\
\alpha_{h_{4}} & \alpha_{h_{6}} & \cdots & \alpha_{h_{2}} & \alpha_{h_{3}} & \alpha_{h_{5}} & \cdots & \alpha_{h_{1}} \\
\alpha_{h_{2 k-1}} & \alpha_{h_{1}} & \cdots & \alpha_{h_{2 k-3}} & \alpha_{h_{0}} & \alpha_{h_{2}} & \cdots & \alpha_{h_{2 k}} \\
\alpha_{h_{2 k-3}} & \alpha_{h_{2 k-1}} & \cdots & \alpha_{h_{2 k-5}} & \alpha_{h_{2 k}} & \alpha_{h_{0}} & \cdots & \alpha_{h_{2 k-2}} \\
\vdots & \vdots & \ddots & \vdots & \vdots & \vdots & \ddots & \vdots \\
\alpha_{h_{1}} & \alpha_{h_{3}} & \cdots & \alpha_{h_{2 k-1}} & \alpha_{h_{4}} & \alpha_{h_{6}} & \cdots & \alpha_{h_{2}}
\end{array}\right) .
$$

Hence $\sigma(v)$ is of the form

$$
\left(\begin{array}{ll}
A & B \\
D & A
\end{array}\right)
$$

where $A, B$ and $D$ are circulant matrices.

Choose an element of $v$ such that $v=\sum \alpha_{i} h_{i}$ where only one of $\alpha_{2 i}=1$ and the rest of $\alpha_{2 i}$ are 0 . Then the generating matrix is permutation equivalent to a matrix where $A$ is $I_{k}$ and $B$ and $D$ are circulant matrices. Namely, we get a matrix of the form

$$
\left(\begin{array}{cc}
I_{\frac{n}{2}} & B \\
D & I_{\frac{n}{2}}
\end{array}\right) \text {. }
$$


Theorem 6.1. Let $R$ be a Frobenius ring of characteristic 2 and let $v \in R C_{n}$ with $v=$ $\sum \alpha_{i} h_{i}$ where only one $\alpha_{2 i}=1$ and the rest of $\alpha_{2 i}$ are 0 . If $v_{2 k-i}=v_{i}$ for odd $i$ and $|C|=|R|^{k}$ then $C(v)$ is a self-dual code.

Proof. By the construction, we have that $\sigma(v)$ is of the form

$$
\left(\begin{array}{cc}
I_{k} & B \\
D & I_{k}
\end{array}\right) .
$$

If $v_{2 k-i}=v_{i}$ for odd $i$ then $D=B^{T}$. We have that $|C|=|R|^{k}$. However, the form of the matrix gives that $C$ contains a free code isomorphic to $R^{k}$, namely the code generated by the matrix $\left(I_{k} \mid B\right)$. This means that $C=\left\langle\left(I_{k} \mid B\right)\right\rangle$.

Consider the code generated by the matrix $\left(B^{T} \mid I_{k}\right)$. This code must be $C^{\perp}$. However, this code is contained in $C(v)$ as well, so we have that $C=C^{\perp}$.

Notice that we did not have to determine the cardinality of the code to see that the code was self-dual.

Note that it is certainly more difficult to use this technique to construct self-dual codes with the cyclic group. That is, we had to put more restrictions on $v$ to obtain a self-dual code. This is certainly to be expected since it is fairly difficult to find cyclic self-dual codes.

Moreover, note that a code over $R_{k}$ constructed with this technique is cyclic, which gives that its image under the Gray map is quasi-cyclic of index $2^{k}$.

Example 12. Let $G$ be the cyclic group of order 10 and $v=1+u h+h^{5}+u h^{9} \in R_{1} C_{10}$. Then $C_{v}=\langle\sigma(v), u \sigma(v)\rangle$ is cyclic self-dual code and its image under $\phi_{1}$ is a binary quasi-cyclic self-dual $[20,10,4]$ code of index 2.

We note that this is a standard construction of self-dual codes, namely you take a vector $\mathbf{v}$ and generate a circulant matrix $B$ from it with $B B^{T}=-I_{k}$, with $n=2 k$, and generate the code $\left(I_{k} \mid B\right)$. Hence, we have another of the standard constructions of self-dual codes within our general framework.

We can now use our general construction to produce isodual codes.

Theorem 6.2. Let $R$ be a finite commutative Frobenius ring with characteristic 2. Let $v \in R C_{n}$ with $v=\sum \alpha_{i} h_{i}$ where only one $\alpha_{2 i}=1$ and the rest of $\alpha_{2 i}$ are 0 . If $|C(v)|=|R|^{\frac{n}{2}}$ then $C(v)$ is a formally self-dual code with respect to any weight enumerator.

Proof. If $|C(v)|=|R|^{\frac{n}{2}}$ then $C$ is generated by the matrix $\left(I_{k} \mid B\right)$ where $B$ is a circulant matrix. Then its orthogonal is of the form $\left(B^{T} \mid I_{k}\right)$. Since $B$ is a circulant code, then by permuting the rows and columns of $B$ we can form $B^{T}$. This gives that $C(v)^{\perp}$ is equivalent to $C(V)$ and hence isodual and therefore formally self-dual code with respect to any weight enumerator. 
Example 13. Let $G$ be the cyclic group of order 6 and $v=1+u_{2} h+\left(1+u_{1}+u_{1} u_{2}\right) h^{3}+u_{1} h^{5} \in$ $R_{2} C_{6}$. Then $C_{v}=\left\langle\sigma(v), u_{1} \sigma(v), u_{1} u_{2} \sigma(v)\right\rangle$ is a cyclic formally self-dual code and its image under $\phi_{2}$ is a binary quasi-cyclic self-dual $[24,12,6]$ code of index 4 .

Example 14. Let $G$ be the cyclic group of order 10. The following elements of $R_{2} C_{10}$ generate four inequivalent binary self-dual [40,20,8] codes:

\begin{tabular}{|c|c|c|}
\hline$i$ & $v_{i} \in R_{2} C_{10}$ & $\mid$ Aut $\left(C_{v_{i}}\right) \mid$ \\
\hline \hline 1 & $1+u_{1}\left(h+h^{9}\right)+u_{2}\left(h^{3}+h^{7}\right)+h^{5}$ & $2^{16} \cdot 3^{3} \cdot 5^{2}$ \\
\hline 2 & $1+u_{1}\left(h+h^{9}\right)+u_{2}\left(h^{3}+h^{7}\right)+\left(u_{1} u_{2}+1\right) h^{5}$ & $2^{14} \cdot 3 \cdot 5$ \\
\hline 4 & $1+u_{1}\left(h+h^{9}\right)+u_{2}\left(h^{3}+h^{7}\right)+\left(u_{2}+1\right) h^{5}$ & $2^{14} \cdot 3 \cdot 5$ \\
\hline 5 & $1+u_{1}\left(h+h^{9}\right)+u_{2}\left(h^{3}+h^{7}\right)+\left(u_{1}+u_{1}+1\right) h^{5}$ & $2^{16} \cdot 3^{3} \cdot 5^{2}$ \\
\hline
\end{tabular}

\section{Quasi- $G$ Codes}

In this section, we make a generalization of the notion of a quasi-cyclic group. In general, quasi-cyclic groups are more difficult to handle that cyclic codes because they do not have a canonical representation in an algebraic setting the way that cyclic codes do. In [6], a ring was developed with a Gray map that could be used to describe certain families of quasicyclic groups. That same ring can be used in this setting to construct quasi- $G$ codes which we shall describe below. Self-dual codes over these rings were studied in [7].

Let $G$ be a finite group of order $n$ and $R$ a finite Frobenius commutative ring. Let $D$ be a code in $R^{s n}$ where the coordinates can be partitioned into $n$ sets of size $s$ where each set is assigned an element of $G$. If the code $D$ is held invariant by the action of multiplying the coordinate set marker by every element of $G$ then the code $D$ is called a quasi-group code of index $s$.

We now describe a family of rings to construct quasi- $G$ codes.

Let $p_{1}, p_{2}, \ldots, p_{t}$ be prime numbers with $t \geq 0$ and $p_{i} \neq p_{j}$ if $i \neq j$. Define $\Delta$ to be $\Delta=p_{1}^{k_{1}} p_{2}^{k_{2}} \cdots p_{t}^{k_{t}}$, for some $k_{i} \geq 1, i=1, \ldots, t$.

The ring is defined as follows:

$$
R_{q, \Delta}=\mathbb{F}_{q}\left[u_{p_{1}, 1}, \ldots, u_{p_{1}, k_{1}}, u_{p_{2}, 1} \ldots, u_{p_{2}, k_{2}}, \ldots, u_{p_{t}, k_{t}}\right] /\left\langle u_{p_{i}, j}^{p_{i}}=0\right\rangle,
$$

where the indeterminates $\left\{u_{p_{i}, j}\right\}_{\left(1 \leq i \leq t, 1 \leq j \leq k_{i}\right)}$ commute.

Let $i \in\{1, \cdots, t\}, j \in\left\{1, \cdots, k_{i}\right\}$. Take the set of exponents $J_{i}=\left\{0,1, \ldots, p_{i}-1\right\}$ for the indeterminant $u_{p_{i}, j}$. For $\alpha_{i} \in J_{i}^{k_{i}}$ denote $u_{p_{i}, 1}^{\alpha_{i}, 1} \cdots u_{p_{i}, k_{i}}^{\alpha_{i}, k_{i}}$ by $u_{i}^{\alpha_{i}}$. For a monomial $u_{1}^{\alpha_{1}} \cdots u_{t}^{\alpha_{t}}$ in $R_{q, \Delta}$ write $u^{\alpha}$, where $\alpha=\left(\alpha_{1}, \ldots, \alpha_{t}\right) \in J_{1}^{k_{1}} \times \cdots \times J_{t}^{k_{t}}$.

Let $J=J_{1}^{k_{1}} \times \cdots \times J_{t}^{k_{t}}$. Any element $c$ in $R_{q, \Delta}$ can be written as

$$
c=\sum_{\alpha \in J} c_{\alpha} u^{\alpha}=\sum_{\alpha \in J} c_{\alpha} u_{p_{1}, 1}^{\alpha_{1}, 1} \cdots u_{p_{1}, k_{1}}^{\alpha_{1}, k_{1}} \cdots u_{p_{t}, 1}^{\alpha_{t}, 1} \cdots u_{p_{t}, k_{t}}^{\alpha_{t}, k_{t}}
$$


with $c_{\alpha} \in \mathbb{F}_{q}$.

It is immediate that $R_{q, \Delta}$ is a commutative ring with $\left|R_{q, \Delta}\right|=q^{p_{1}^{k_{1}} p_{2}^{k_{2}} \cdots p_{t}^{k_{t}}}=q^{\Delta}$.

Next we define a Gray map on this ring. We will consider the elements in $R_{q, \Delta}$ as $q$-ary vectors of $\Delta$ coordinates. Order the elements of $A_{\Delta}$ lexicographically and use this ordering to label the coordinate positions of $\mathbb{F}_{q}^{\Delta}$. Define the Gray map $\Psi_{\Delta}: A_{\Delta} \rightarrow \mathbb{F}_{q}^{\Delta}$ as follows:

$$
\Psi_{\Delta}(a)_{b}= \begin{cases}1 & \text { if } \widehat{b} \subseteq\{\widehat{a} \cup 1\} \\ 0 & \text { otherwise }\end{cases}
$$

where $\Psi_{\Delta}(a)_{b}$ indicates the coordinate of $\Psi_{\Delta}(a)$ corresponding to the position of the element $b \in A_{\Delta}$ with the defined ordering.

It follows that $\Psi_{\Delta}(a)_{b}$ is 1 if each indeterminate $u_{p_{i}, j}$ in the monomial $b$ with non-zero exponent is also in the monomial $a$ with the same exponent. In other words, it is 1 when $\widehat{b}$ is a subset of $\widehat{a}$. In order to consider all the subsets of $\widehat{a}$, we also add the empty subset that is given when $b=1$; that is we compare $\widehat{b}$ to $\widehat{a} \cup 1$.

Finally, we extend $\Psi_{\Delta}$ linearly for all elements of $R_{q, \Delta}$. Then $\Psi_{\Delta}$ is a Gray map from $R_{q, \Delta}$ to $\mathbb{F}_{q}^{\Delta}$. Note that the ring $R_{k}$ is $R_{2,2^{k}}$ in this setting.

Theorem 7.1. Let $C$ be a code in $R_{q, \Delta}{ }^{n}$ for a finite group $G$, that is $C$ is a $G$-code. Then $\Psi_{\Delta}(C)$ is a quasi-G code of length $n \Delta$ of index $\Delta$ in $\mathbb{F}_{q}^{\Delta n}$.

Proof. Let $\mathbf{v} \in C$. If $g$ is an element of the group $G$ then $g$ acts on the set of $\Delta$ coordinates corresponding to the element $v_{i} \in R_{q, \Delta}$, that is on $\Psi_{\Delta}\left(v_{i}\right)$ and sends them to the coordinates corresponding to $\Psi_{\Delta}\left(g v_{i}\right)$. Therefore, the image is a quasi- $G$ group of index $\Delta$.

An identical proof gives the following.

Theorem 7.2. Let $C$ be a quasi- $G$ code of length $n$ and of index $k$ over $R_{q, \Delta}$ for a finite group $G$, that is $C$ is a $G$-code. Then $\Psi_{\Delta}(C)$ is a quasi-G code of length $n \Delta$ of index $k \Delta$ in $\mathbb{F}_{q}^{\Delta n}$.

Example 15. In Example 5, it is shown that if $v=u\left(b+a b+a c+b c^{2}\right)+\left(b c+b c^{2}\right)+$ $(1+u)\left(c^{2}+a b c^{2}\right) \in R_{1} A_{4}$, then, $C_{v}$ is a self-dual code of length 12 over $R_{1}$. This gives that $\phi_{1}(C)$ is the length 24 binary Golay code. It follows then that the binary $[24,12,8]$ Golay code is a quasi-Alternating group of order 4 code with index 2.

Example 16. In Example 10, it is shown that if $v=1+a\left(\left(u+u b+u b^{2}+b^{3}\right)+(u+b+\right.$ $\left.\left.b^{2}+u b^{3}\right)\left(h+h^{4}\right)+\left(1+b+u b^{3}\right)\left(h^{2}+h^{3}\right)\right) \in R_{1} C_{5} D_{8}$, then $C_{v}=\langle\sigma(v), u \sigma(v)\rangle$ is the binary $[80.40,12]$ self-dual code under $\phi_{1}$. Therefore this code is a quasi- $C_{5} D_{8}$ code of index 2.

Example 17. In Example 14, it is shown that inequivalent binary self-dual [40,20,8] codes are constructed from $R_{2} C_{10}$. It follows that these four codes are quasi-cyclic code of index 4 . 


\section{Conclusion}

In this paper, we have considered a very broad generalization of the notion of cyclic codes by examining codes that are ideals in a group ring, calling these codes $G$-codes. Similar theorems to the standard results on cyclic codes have been attained. For instance, we have shown that the dual of $G$-code is again a $G$-code. This natural algebraic setting allows for canonical constructions of families of codes and for ensuring that the automorphism group of a code contains a given group. We used the Chinese Remainder Theorem to construct $G$-codes over arbitrary rings from $G$-codes over local rings.

Based on some previously known constructions for group algebras, we have given generalized constructions of self-dual codes in this setting for codes over a Frobenius ring. We have given the form of the generator matrix for codes in this construction for various groups. We have shown precisely which groups will give the binary Golay self-dual code in this setting, namely the Golay code is a $G$-code for the following groups: $S_{4}, D_{24}, C_{3} \times D_{8}, C_{2} \times A_{4}$ and $\left(C_{6} \times C_{2}\right) \rtimes C_{2}$.

The ring family of rings $R_{k}$ has also been used to construct interesting binary self-dual codes via the canonical Gray map. This allows for a construction of longer self-dual and formally self-dual binary codes.

The notion of quasi-cyclic codes has also been generalized and we have used the family of rings $R_{q, \Delta}$ to produce families of quasi- $G$ codes over a finite Frobenius ring. We have given several examples of binary quasi- $G$ codes for various groups.

The fundamental open question is to determine which codes are $G$-codes for a finite group $G$. That is, given an arbitrary code over a ring $R$, for which groups $G$ can $C$ be seen as an ideal in $R G$. Since even the case when $G$ is the cyclic group (cyclic codes) remains a large area of research, it seems that there is a great deal of work to be done in this area. More computationally, it should be determined which self-dual codes (especially optimal self-dual codes) can be constructed via the methods described in this paper. Within this framework, these computational techniques can be extended to numerous families of rings and numerous groups.

\section{References}

[1] Bernhardt, F., Landrock, P., Manz, O., The extended Golay codes considered as ideals, J. Combin. Theory Ser. A, 55, no. 2., 1990, 235 - 246.

[2] Borello, M., The automorphism group of a self-dual $[72,36,16]$ code is not an elementary abelian group of order 8, Finite Fields Appl. 25, 2014, 1 - 7.

[3] Bouyuklieva, S., On the automorphism of order 2 with fixed points for the extremal self-dual codes of length 24m, Des. Codes Cryptogr., 25, no. 1, 2002, 5 - 13. 
[4] Bouyuklieva, S., O'Brien, E.A. Willems, W., The automorphism group of a binary self-dual doubly-even $[72,36,16]$ code is solvable, IEEE Trans. Inform. Theory, 52, 2006, $4244-4248$.

[5] Dougherty, S.T., Algebraic Coding Theory Over Finite Commutative Rings, SpringerVerlag, Springer Briefs in Mathematics (ISBN 978-3-319-59805-5), 2017.

[6] Dougherty, S.T., Fernandez-Cordoba, D., Ten-Valls, R., Quasi-Cyclic Codes as Cyclic Codes over a Family of Local Rings, Finite Fields and Their Apps., 40, 2016, 138 149 .

[7] Dougherty, S.T., Kaya, A., Salutrk, E., Constructions of Self-Dual Codes and Formally Self-Dual Codes over Rings, AAECC, DOI 10.1007/s00s00-016-0288-5, 2016.

[8] Dougherty, S.T., Kim, J.L., Solé, P., Open Problems in Coding Theory, Contemporary Mathematics, 634, 2015, 79 - 99.

[9] Dougherty, S.T., Yildiz, B., Karadeniz, S., Codes over $R_{k}$, Gray maps and their Binary Images, with Finite Fields and their Applications, 17, no. 3, 2011, 205 - 219.

[10] Dougherty, S.T., Yildiz, B., Karadeniz, S., Cyclic Codes over $R_{k}$, Designs, Codes and Cryptography, 63, no. 1., 2012, 113 - 126.

[11] Dougherty, S.T., Yildiz, B., Karadeniz, S., Self-dual codes over $R_{k}$ and binary self-dual codes. Eur. J. Pure Appl. Math. 6, no. 1, 2013, 89 - 106.

[12] The GAP Group, GAP - Groups, Algorithms and Programming, Version 4.4, 2006 (http:/www.gap-system.org).

[13] Hurley, T., Group Rings and Rings of Matrices, Int. Jour. Pure and Appl. Math,, 31, no. 3, 2006, 319 - 335 .

[14] Lam, C. W. H.; Thiel, L.; Swiercz, S. The nonexistence of finite projective planes of order 10. Canad. J. Math. 41, no. 6, 1989, 1117 - 1123.

[15] MacWilliams, F. J., Binary codes which are ideals in the group algebra of an Abelian group. Bell System Tech. J. 49, 1970, 987 - 1011.

[16] MacWilliams, F.J., Codes and ideals in group algebras. 1969 Combinatorial Mathematics and its Applications (Proc. Conf., Univ. North Carolina, Chapel Hill, N.C., 1967) 317 - 328. Univ. North Carolina Press, Chapel Hill, N.C.

[17] McLoughlin, I., Dihedral codes, Ph.D. thesis, National University of Ireland, Galway, 2009. 
[18] McLoughlin, I., A group ring construction of the [48, 24, 12] Type II linear block code, Des. Codes Cryptogr., 63, no. 1, 2012, 29 - 41.

[19] McLoughlin, I., Hurley, T., A group ring construction of the extended binary Golay code, IEEE Trans. Inform. Theory, 54, no. 9, 2008, 4381 - 4383.

[20] Nebe, G., An extremal [72,36,16] binary code has no automorphism group containing $\mathbb{Z}_{2} \times \mathbb{Z}_{4}, Q_{8}$ or $\mathbb{Z}_{10}$, Finite Fields Appl., 18, no. 3, 2012, 563 - 566.

[21] O'Brien, E.A., Willems, W., On the automorphism group of a binary self-dual doublyeven [72,36, 16] code, IEEE Trans. Inform. Theory, 57, no.7, 2011, 4445 - 4451.

[22] Yankov, N., A putative doubly-even [72,36, 16] code does not have an automorphism of order 9, IEEE Trans. Inform. Theory, 58, no. 1, 2012, 159 - 163. 